\title{
Optimal Recombination and Adaptive Restarts Improve GA Performance on the Asymmetric TSP
}

\author{
Anton Eremeev ${ }^{*}$ \\ Institute of Scientific Information for Social Sciences RAS \\ Moscow, Russia \\ Dostoevsky Omsk State University \\ Omsk, Russia
}

\author{
Yulia Kovalenko \\ Institute of Scientific Information for Social Sciences RAS \\ Moscow, Russia \\ Dostoevsky Omsk State University \\ Omsk, Russia
}

\begin{abstract}
We propose a new genetic algorithm (GA) with optimal recombination and adaptive restarts for the asymmetric travelling salesman problem (ATSP). The optimal recombination problem (ORP) is solved in a crossover operator based on a new exact algorithm that solves the ATSP on cubic digraphs. We use an adaptive restart rule based on the Schnabel census estimate. A computational experiment on the known benchmark instances shows that the proposed algorithm yields results competitive to those of state-of-the-art algorithms for the ATSP and confirms that the ORP may be used successfully in genetic algorithms.

This abstract for the Hot-off-the-Press track of GECCO 2021 summarizes work that has appeared in Eremeev A.V., Kovalenko Yu.V. A memetic algorithm with optimal recombination for the asymmetric traveling salesman problem. Memetic Computing 12, 23-36 (2020).
\end{abstract}

\section{CCS CONCEPTS}

- Theory of computation $\rightarrow$ Routing and network design problems; • Computing methodologies $\rightarrow$ Randomized search; Genetic algorithms.

\section{KEYWORDS}

Optimal recombination, Local search, Restart rule, Computational experiment

\section{ACM Reference Format:}

Anton Eremeev and Yulia Kovalenko. 2021. Optimal Recombination and Adaptive Restarts Improve GA Performance on the Asymmetric TSP. In 2021 Genetic and Evolutionary Computation Conference Companion (GECCO '21 Companion), fuly 10-14, 2021, Lille, France. ACM, New York, NY, USA, 2 pages. https://doi.org/10.1145/3449726.3462729

\section{SUMMARY OF OUR RESULTS}

Genetic algorithms (GAs) are optimization methods inspired by natural biological evolution. A GA starts with an initial population, and goes from the current population to the next one applying selection, crossover (recombination) and mutation. The performance of a GA depends on the choice of the crossover operator, where the

*Both authors contributed equally to this research.

Permission to make digital or hard copies of part or all of this work for personal or classroom use is granted without fee provided that copies are not made or distributed for profit or commercial advantage and that copies bear this notice and the full citation on the first page. Copyrights for third-party components of this work must be honored For all other uses, contact the owner/author(s).

GECCO '21 Companion, fuly 10-14, 2021, Lille, France

(c) 2021 Copyright held by the owner/author(s)

ACM ISBN 978-1-4503-8351-6/21/07.

https://doi.org/10.1145/3449726.3462729 components of parent solutions are combined to build new solutions. A supplementary problem that emerges in some crossover operators is called the Optimal Recombination Problem (ORP) [1, 6]. Given two feasible parent solutions, the ORP consists in finding the best possible offspring, where an allele of each gene is copied from one of the alleles of this gene in the parent solutions. Such recombination operators are called (strictly) gene transmitting [10]. Crossover operators solving the ORP exactly are usually called optimized crossover operators.

The first GA using ORP for the Maximum Independent Set problem appeared in the paper of Agarwal, Orlin and Tai [1]. Further results, e.g. $[3,13,15]$, added more experimental support to expediency of solving optimal recombination problems in crossover operators (in particular for permutation problems).

The Travelling Salesman Problem (TSP) is one of the classical combinatorial optimization problems [8]. Given a complete digraph $G$ with the set of vertices $V=\left\{v_{1}, \ldots, v_{n}\right\}$, the set of $\operatorname{arcs} A=$ $\left\{\left(v_{i}, v_{j}\right): v_{i}, v_{j} \in V, i \neq j\right\}$ and arc weights (lengths) $c\left(v_{i}, v_{j}\right) \geq 0$ of each $\operatorname{arc}\left(v_{i}, v_{j}\right) \in A$, the TSP asks for a Hamiltonian circuit (a tour) with the minimum total length. If $c\left(v_{i}, v_{j}\right) \neq c\left(v_{j}, v_{i}\right)$ for at least one pair $v_{i}, v_{j}$ then the TSP is called the Asymmetric Travelling Salesman Problem (ATSP).

We propose an ORP-based GA with adaptive restarts for the ATSP. The initial population is built using the greedy constructive heuristic of W. Zhang [16] (first solves an assignment problem, then applies the Karp patching) and the well known arbitrary insertion method [15]. The GA is based on the steady state replacement. A new mutation operator making random jumps in 3-opt or 4-opt neighborhoods is applied to parent solutions before recombination.

We use vectors of adjacencies for encoding tours of the ATSP. In these vectors, the immediate predecessor is indicated for each vertex. In the case of adjacency-based representation, the ORP aims at finding a travelling salesman's tour of minimum length among the circuits composed only of the arcs that are present in the parents, and contain all arcs common to both parents. The ORP for the ATSP is NP-hard [6], and may be considered as Forced ATSP on a digraph with bounded vertex degree, where the forced arcs correspond to common parent arcs. Then the ORP can be solved in $O\left(2^{d / 4}\right)$ time, where $d \leq 2 n$ is the number of arcs which are present in one of the parents only. Here we use a special adaptation of the recursive algorithm of D. Eppstein[5], originally proposed for listing all Hamiltonian cycles in a graph of degree three.

In our GA, individuals of the initial and the final populations are improved by the 3-opt local search with the well-known "don't look bits" and "first improving move" strategies. Moreover, the optimized crossover may be considered as a best-improving move in 
the neighborhood defined by two (parent) solutions. The balance between different local search operators (3-opt on the initial and the final population and the optimized crossover during the evolution stage) is based on a GA restarting rule. In this paper, we consider two restarting rules. The first approach uses the classic restart rule, where the GA gets restarted as soon as the current iteration number becomes twice the iteration number when the best incumbent was found (denoted $G A_{C L}$ ). The second approach [7] is based on the Schnabel census method from biometrics, coupled with the maximum likelihood principle. This rule restarts the algorithm when the population diversity becomes so small that new solutions are unlikely to be found. The GA with this rule is denoted $G A_{S C}$.

We compare our ORP-based GA with various state-of-the-art approaches known for the ATSP:

- Memetic algorithm with Strategic Arc Crossover (SAX) and Recursive Arc Insertion (RAI) for local improvements $\left(M A_{S A X / R A I}\right)$ of Buriol et al. [2],

- GA with Generalized Asymmetric Partition Crossover (GAPX) and 3-opt local optimization ( $\left.G A_{G A P X}\right)$ of Tinós et al. [13],

- GA with Edge Assembly Crossover (EAX) and 3-opt local optimization of Nagata and Soler [9].

The listed GAs use adjacency-based crossover operators and apply a local search on GA iterations. The GAPX crossover from [13] produces a greedy approximate solution to the ORP.

An experimental evaluation was performed on 27 asymmetric instances from the TSPLIB library (series $\mathrm{ftv}$, $\mathrm{ft}$, and $\mathrm{rbg}$, and individual problems br17, p43, ry48p, kro124p) [11]. We arranged the experiment so that both our GA using the restart rule based on the Schnabel census method $G A_{S C}$ and an algorithm from the literature were given approximately equivalent CPU resource (scaling factors for the CPU-time limit are chosen on the basis a rough comparison of computers by performance table [4] and the CPU clock rates). We compared the frequencies of finding an optimum.

$M A_{S A X / R A I}$ [2] is one of the most well-known heuristics for the ATSP. Initially we compare $G A_{S C}$ with $M A_{S A X / R A I}$. On each instance, $G A_{S C}$ finds optima in at least $99 \%$ of the runs, and $M A_{S A X / R A I}$ in $84 \%$ of the runs on average. In 14 out of 27 instances, $G A_{S C}$ finds an optimum more often than $M A_{S A X / R A I}$ (in all 14 cases the difference between the frequencies is statistically significant at level $p \leq 0.05$ ). Both algorithms demonstrate $100 \%$ frequency of obtaining an optimum on 13 problems.

$G A_{G A P X}$ [13] presents $100 \%$ frequency of obtaining an optimum on all of 19 considered asymmetric instances from TSPLIB, given very large CPU time. $G A_{S C}$ also showed $100 \%$ frequency of success under the CPU resource similar to [13].

$G A_{E A X}$ [9] is one of the fastest metaheuristics, known for the ATSP. The average frequency of obtaining an optimum for $G A_{S C}$ is 0.972 , while that of $G A_{E A X}$ is 0.953 . In 11 out of 27 instances, $G A_{S C}$ finds an optimum more often than $G A_{E A X}$ (in 3 cases among these the difference between the frequencies is statistically significant). $G A_{E A X}$ outperforms $G_{S C}$ on four instances, but the difference between the frequencies of finding an optimum is not statistically significant. We also tested $G A_{S C}$ on 128 instances ( $n$ is ranged from 64 to 315) from [12] and compared with $G A_{E A X}$.

Effects of Restarting Rules and Optimal Recombination. Preliminary computational experiments showed that by restarting our GA, we avoid localization of the search and restore the population diversity, which leads to better results than in the case of no restarts, given the same amount of time.

We also compared two versions of our algorithm with restarts under the same conditions as in the experiment with $G A_{E A X}$. The $G A_{S C}$ demonstrated better results than the $G A_{C L}$ on 12 instances, and the difference between the frequencies was statistically significant in 5 cases. $G A_{C L}$ slightly outperforms $G A_{S C}$ on 4 instances, but the difference was statistically significant only in one case.

The optimized crossover operator, which solves the ORP exactly, may be considered as a deterministic "direct descendant" of the Directed Edge Crossover (DEC) [14]. The computational evaluation clearly shows an advantage of the ORP-based crossover over its randomized prototype DEC in our GA.

\section{Conclusion}

The proposed genetic algorithm for the ATSP uses the steady-state population management strategy, adjacency-based solutions representation, optimal recombination, local search and a restarting rule based on Schnabel census. The results of computational experiments show that the proposed GA yields results competitive or superior to those of other well-known algorithms.

\section{ACKNOWLEDGMENTS}

Supported by the Russian Science Foundation grant 17-18-01536.

\section{REFERENCES}

[1] C.C. Agarwal, J.B. Orlin, and R.P. Tai. 1995. Optimized crossover for the independent set problem. working paper no. 3787-95. Massachusetts Institute of Technology.

[2] L. S. Buriol, P. M. Franca, and P. Moscato. 2004. A new memetic algorithm for the asymmetric traveling salesman problem. fournal of Heuristics 10 (2004), 483-506.

[3] F. Chicano, G. Ochoa, D. Whitley, and R. Tinos. 2019. Quasi-optimal recombination operator. In Evolutionary Computation in Combinatorial Optimization, 2019, LNCS, Vol. 11452. 131-146.

[4] J. J. Dongarra. 2014. Performance of Various Computers Using Standard Linear Equations Software. Tech. Rep. CS-89-85. University of Manchester. $110 \mathrm{p}$.

[5] D. Eppstein. 2007. The Traveling Salesman Problem for Cubic Graphs. Fournal of Graph Algorithms and Applications 11, 1 (2007).

[6] A.V. Eremeev. 2011. On Complexity of the Optimal Recombination for the Travelling Salesman Problem. In Evolutionary Computation in Combinatorial Optimization. Springer Berlin Heidelberg, 215-225.

[7] A. V. Eremeev. 2019. A Restarting Rule Based on the Schnabel Census for Genetic Algorithms. In Learning and Intelligent Optimization, LNCS, Roberto Battiti, Mauro Brunato, Ilias Kotsireas, and Panos M. Pardalos (Eds.), Vol. 11353. Springer International Publishing, Cham, 337-351.

[8] M. R. Garey and D. S. Johnson. 1979. Computers and Intractability. A Guide to the Theory of NP-completeness. W. H. Freeman and Company, San Francisco.

[9] Y. Nagata and D. Soler. 2012. A new genetic algorithm for the asymmetric TSP. Expert Syst. with Applications 10 (2012), 8947-8953.

[10] N. J. Radcliffe. 1994. The Algebra of Genetic Algorithms. Annals of Mathematics and Artificial Intelligence 10, 4 (1994), 339-384.

[11] G. Reinelt. 1991. TSPLIB - A Traveling Salesman Problem Library. ORSA fournal on Computing 3, 4 (1991), 376-384.

[12] D. Soler, E. Martinez, and Mico J.C. 2008. A transformation for the mixed general routing problem with turn penalties. Fournal of the Operational Research Society 59 (2008), 540-547.

[13] R. Tinós, D. Whitley, and G. Ochoa. 2014. Generalized Asymmetric Partition Crossover (GAPX) for the Asymmetric TSP. In The 2014 Annual Conference on Genetic and Evolutionary Computation. ACM New York, NY, 501-508.

[14] D. Whitley, T. Starkweather, and D. Shaner. 1991. The Traveling Salesman and Sequence Scheduling: Quality Solutions Using Genetic Edge Recombination. In Handbook of Genetic Algorithms. Van Nostrand Reinhold, NY, 350-372.

[15] M. Yagiura and T. Ibaraki. 1996. The use of dynamic programming in genetic algorithms for permutation problems. Eur. Four. Oper. Res. 92 (1996), 387-401.

[16] W. Zhang. 2000. Depth-First Branch-and-Bound versus Local Search: A Case Study. In 17th National Conf. on Artificial Intelligence. Austin, TX, 930-935. 